\title{
Ajuste dos simuladores de dados meteorológicos do modelo EPIC para diferentes locais do Estado de São Paulo'
}

\author{
Angélica G. Picini ${ }^{2}$, Márcio de M. Valeriano ${ }^{3}$, Francisco Lombardi Neto ${ }^{4} \&$ Jurandir Zullo Júnior ${ }^{5}$
}

\footnotetext{
1 Parte da Tese de Doutorado do primeiro autor, apresentada à UNICAMP

2 Instituto Nacional de Pesquisas Espaciais, Divisão de Satélites e Sistemas Ambientais - CPTEC. Rod. Presidente Dutra Km 40, CEP 12630-000, Cachoeira Paulista, SP. Fone: (12) 3186-9324. E-mail: angelica@cptec.inpe.br (Foto)

${ }^{3}$ Instituto Nacional de Pesquisas Espaciais - DSR/OBT. CEP 12227-010. São José dos Campos, SP. Fone: (12) 3945 6499. Fone: (12) 3945-6499. E-mail: valerian@ltid.inpe.br

${ }^{4}$ Instituto Agronômico de Campinas, Centro de Solos e Recursos Agroambientais, CP:28, CEP 13020-902, Campinas, SP. Fone: (19) 3241-5188

5 CEPAGRI/UNICAMP - Cidade Universitária "Zeferino Vaz", CEP 13083-970. Campinas, SP. Fone: (19) 3788-2260. E-mail: jurandir@cpa.unicamp.br
}

Protocolo 160 - 23/10/2003 - Aprovado em 28/6/2004

\begin{abstract}
Resumo: O modelo EPIC (Erosion Productivity Impact Calculator) foi desenvolvido para simular o efeito de estratégias de manejos agrícolas em recursos hídricos e produtividade de solos. No presente estudo, o desempenho dos simuladores de dados meteorológicos foi avaliado para localidades do Estado de São Paulo (Campinas, Jaú, Manduri, Mococa, Pindorama e Ubatuba), incluindo: a Cadeia de Markov para probabilidades de seqüências de dias úmidos e secos; a equação de temperatura máxima ajustada para dias úmidos e a equação de radiação solar, também ajustada para dias úmidos. A partir dos testes e ajustes dos modelos com os dados históricos, verificou-se o melhor desempenho para o modelo de probabilidade de seqüências de dias secos, considerando-se o dia anterior úmido - $P(W \mid D)$ em relação ao modelo de probabilidade de seqüências para dois dias úmidos consecutivos - $\mathrm{P}(\mathrm{W} \mid \mathrm{W})$. Os modelos de temperatura máxima do ar e de radiação solar apresentaram bom desempenho nos testes. Para todos os modelos, os melhores resultados foram obtidos a partir dos ajustes para cada local, quando comparados às estimativas obtidas pelos valores originais propostos na concepção do EPIC. Ubatuba apresentou comportamento distinto.
\end{abstract}

Palavras-chave: clima, modelos de erosão, probabilidade

\section{Adjustment of meteorological data simulators of the EPIC model for different localities in the State of São Paulo, Brazil}

\begin{abstract}
EPIC (Erosion Productivity Impact Calculator) model has been developed to simulate the effect of agricultural management strategies of water resources and soil productivity. In this study, the performance of meteorological data simulators were evaluated for six (Campinas, Jaú, Manduri, Mococa, Pindorama and Ubatuba) localities in the State of São Paulo, Brazil, which include the Markov chain probabilities for the sequences of wet and dry days; the maximum air temperature model adjusted for wet days; and the solar radiation model also adjusted for wet days. From tests and adjustments of the models using the historical data for each locality, the best performance for the model of probabilities for dry days sequence has been found, taking into account the last wet day - P(W|D) in relation to the model of probabilities of two consecutive wet days sequence - $\mathrm{P}(\mathrm{W} \mid \mathrm{W})$. The maximum air temperature and solar radiation models have presented good performance in tests after the adjustment for each locality. For all models, the best results have been obtained from the local adjustments, when compared to the original model conceived for EPIC. Model results for Ubatuba data were distinct from the other sites.
\end{abstract}

Key words: climate, erosion models, probability 


\section{INTRODUÇÃO}

A Equação Universal de Perda de Solo - EUPS (Wischmeier \& Smith, 1978) constitui-se a primeira aproximação matemática para simular a perda de solo em função da erosão hídrica; no entanto, é um modelo em que não se inclui a estimativa do material depositado que ocorre nos perfis côncavos e convexos das vertentes ou mesmo a produção de sedimentos dos setores à montante do segmento considerado. Mesmo assim, o modelo acima citado tem sido amplamente utilizado em estudos agrícolas. Posterior à difusão da EUPS, Sharpley \& Williams (1990) desenvolveram o modelo Erosion Productivity Impact Calculator (EPIC), que se trata de um conjunto de modelos matemáticos com a finalidade de quantificar a relação entre a erosão e a produtividade das culturas. O EPIC consiste, portanto, em equações baseadas em componentes físicos para simular a erosão e avaliar a sustentabilidade da capacidade produtiva do solo (Sharpley \& Williams, 1990; Rao et al., 2000).

Aplicado em sua forma mais ampla, os componentes considerados em sua análise são: hidrologia, clima, erosão do solo, nutrientes, práticas de cultivo, temperatura do solo, aspectos econômicos e espécie cultivada totalizando, assim, diferentes módulos de processamento dos componentes citados. Todos os módulos do EPIC interagem entre si, com uma concentração de interações dos diferentes módulos em torno do módulo clima.

Sharpley \& Williams (1990) discutiram a possibilidade de desenvolvimento e refinamento dos componentes individuais dos simuladores do EPIC, enfatizando a possibilidade de seu ajuste. Trabalhos como os de Stockle et al. (1992), Cooter \& Dhakhwa (1995) e Easterling et al. (1996), entre outros, são exemplos de adaptações dos simuladores de dados meteorológicos do EPIC.

Os simuladores de dados meteorológicos do EPIC estão incluídos no módulo WXGEN (Weather Generator), desenvolvido para gerar dados diários com as mesmas características estatísticas dos dados observados. São encontrados, nesse módulo, os modelos de simulação de precipitação, de temperatura máxima e mínima do ar, de vento (velocidade e direção) e de umidade relativa (Richardson \& Nicks, 1990). As variáveis climáticas de precipitação, de temperatura e de radiação solar, são utilizadas para simular o escoamento superficial, a erosão do solo e as condições ambientais do sistema solo-planta-água. De maneira análoga, outros modelos (tais como os modelos hidrológicos e de crescimento da cultura, por exemplo) dependem, inicialmente, do ajuste dos simuladores de clima.

O modelo para obtenção de probabilidade de dias secos ou úmidos apresentado no EPIC, consiste em uma Cadeia de Markov de primeira ordem (Nicks, 1974). A Cadeia de Markov é formulada em função da ocorrência ou não de chuva em determinado dia, em vez da quantidade de chuva ou de qualquer outra observação meteorológica (Gabriel \& Neumann, 1962). Numa cadeia de Markov de primeira ordem, a probabilidade de chuva em um dia específico depende somente do estado do dia anterior - seco ou chuvoso. Neste caso, trata-se de um tipo de modelagem que descreve o sistema como um processo caracterizado pelos seus estados e pela dinâmica de sua alternância (Sansigolo, 1988). As estimativas dessas probabilidades são, portanto, as proporções de ocorrência de precipitação observadas, dadas as condições dos dias antecedentes. Os parâmetros a serem estimados são, então, as probabilidades de transição $\mathrm{p} /(\mathrm{t})$, ou seja, as probabilidades de chuva no dia (t) condicionais ao estado i (0/1) do dia (t-1).

No caso da temperatura do ar e da radiação solar, Virgens Filho \& Cataneo (1999) discutem que, além da importância da radiação solar nas características climáticas das regiões, o conhecimento de sua incidência em determinado local é fundamental para estudos agroclimatológicos e para pesquisas visando aproveitamento da energia solar. Para os geradores de temperatura do ar e de radiação solar utilizados no EPIC, Richardson (1981) apresentou um modelo que simula esses dois parâmetros correlacionados à chuva. A dependência estrutural das temperaturas diárias máxima e mínima em relação à radiação solar, foram descritas no trabalho de Richardson (1982).

Desta forma, verifica-se a necessidade de análise desses simuladores, uma vez que esses referidos modelos são considerados parte primordial entre os demais módulos do EPIC, visto que abrangem processos dinâmicos de erosão dos solos, produção de culturas e outros fatores, como custos, práticas de cultivo, entre outros. Os modelos de simulação, uma vez ajustados e testados, podem ser utilizados para preencher lacunas existentes nas séries de informações registradas em estações hidrológicas ou meteorológicas. Entretanto, uma das maiores dificuldades na aplicação desses modelos é a obtenção de dados de entrada confiáveis, entre eles os meteorológicos, para a realização de seu ajuste.

Embora testados e aplicados para diversas localidades em diferentes países, incluindo o Brasil (Sentelhas et al., 2001), não se encontram relatos sobre o ajuste e avaliação da sensibilidade dos simuladores dos dados meteorológicos de entrada do EPIC para São Paulo, que conta com séries históricas de dados em várias localidades.

Considerando-se que os ajustes dos simuladores de dados meteorológicos do EPIC foram feitos em condições climáticas particularizadas, o presente trabalho tem o objetivo de avaliar e verificar a necessidade de ajustes desses simuladores em relação às séries históricas de dados para diferentes condições climáticas do Estado de São Paulo.

\section{MATERIAL E MÉTODOS}

\section{Locais de estudo}

Os locais de estudo selecionados foram: Campinas (lat. $22^{\circ} 54^{\prime}$ S, long. $47^{\circ} 05^{\prime} \mathrm{W}$ e alt. $\left.674 \mathrm{~m}\right)$; Jaú ( $22^{\circ} 17^{\prime} \mathrm{S}, 48^{\circ} 34^{\prime} \mathrm{W}$ e alt. $580 \mathrm{~m})$; Manduri $\left(23^{\circ} 10^{\prime} \mathrm{S}, 49^{\circ} 20^{\prime} \mathrm{W}\right.$ e alt. $\left.589 \mathrm{~m}\right)$; Mococa $\left(21^{\circ} 28^{\prime} \mathrm{S}, 47^{\circ} 01^{\prime} \mathrm{W}\right.$ e alt. $\left.665 \mathrm{~m}\right)$; Pindorama ( $21^{\circ} 13^{\prime} \mathrm{S}, 48^{\circ} 56^{\prime} \mathrm{W}$ e alt. $562 \mathrm{~m})$ e Ubatuba $\left(23^{\circ} 27^{\prime} \mathrm{S}, 45^{\circ} 04^{\prime} \mathrm{W}\right.$ e alt. $\left.8 \mathrm{~m}\right)$.

Segundo a classificação de Köeppen, os locais de estudo podem ser considerados como:

a) Campinas: Cwa; b) Jaú: Cwa; c) Manduri: Cwa/Cfa; d) Mococa: Cwa; e) Pindorama: Aw/Cwa e f) Ubatuba: Af em que: Cwa é caracterizado como clima sub-tropical com inverno seco e verão úmido e quente. Abrange a maior parte do Estado, no centro e leste, com altitudes entre 500 e $700 \mathrm{~m}$; Cfa representa 
o clima subtropical com verão quente e estação seca moderada de inverno. Situa-se na região sudoeste do Estado, permite cultivos de inverno sem irrigação; Aw representa o clima tropical com verão quente e úmido e inverno ameno com estação seca acentuada. Localiza-se no planalto ocidental, com altitudes entre 400 e 500 m, na região norte e noroeste do Estado; Af é caracterizado pelo clima tropical úmido, sem estação seca e relativamente quente $\mathrm{o}$ ano todo e compreende a faixa litorânea do Estado.

Manduri e Pindorama estão em áreas de transição entre os tipos climáticos.

\section{Dados meteorológicos para avaliação dos modelos}

Os dados meteorológicos necessários para avaliação dos simuladores de clima do modelo EPIC, para o Estado de São Paulo, foram fornecidos pelo Centro de Pesquisa e Desenvolvimento de Ecofisiologia e Biofísica do Instituto Agronômico de Campinas (IAC) e o período considerado, de 1962 a 1999, com abrangência de 38 anos de observações diárias para cada elemento. Consideraram-se dados de temperatura máxima do ar $\left({ }^{\circ} \mathrm{C}\right)$, temperatura mínima do ar $\left({ }^{\circ} \mathrm{C}\right)$, chuva $(\mathrm{mm})$ e horas de insolação (h). A temperatura do ar em cada local foi medida em abrigos padronizados segundo a Organização Meteorológica Mundial (OMM), com termômetros de mercúrio em vidro, instalados a $1,70 \mathrm{~m}$ de altura. A chuva foi medida em pluviômetros instalados com a altura de captação de $1,50 \mathrm{~m}$ do solo. Os dados diários de horas de insolação ou brilho solar foram obtidos por heliógrafos tipo Campbell Stokes.

\section{Modelos de probabilidade para dias secos e úmidos do EPIC}

O modelo de probabilidade para dias secos e úmidos utilizado no EPIC foi desenvolvido por Nicks (1974) e é apresentado na Eq. 1.

$$
\mathrm{P}(\mathrm{W} \mid \mathrm{D})=\beta * \mathrm{P}(\mathrm{W})
$$

em que: $\mathrm{P}(\mathrm{W} \mid \mathrm{D})$ é a probabilidade de transição de um dia úmido|seco; $\mathrm{P}(\mathrm{W})$ é a probabilidade de dias úmidos; $\beta$ (beta) é um fator de ajuste dessa transição. Para as condições climáticas testadas, $\beta$ apresenta o valor de 0,75 como satisfatório para a estimativa de $\mathrm{P}(\mathrm{W} \mid \mathrm{D})$ conforme Sharpley \& Williams (1990). De acordo com a metodologia apresentada pelo EPIC considerouse, no presente estudo, que um dia com chuva ou úmido deveria apresentar valores iguais ou superiores a $0,2 \mathrm{~mm}$ (Richardson \& Nicks, 1990).

Considerando-se a transição entre os dois estados, um dia úmido (W) e um dia seco (D), obtiveram-se as seguintes probabilidades: $\mathrm{W} \mid \mathrm{W}$ (úmido|úmido), $\mathrm{W} \mid \mathrm{D}$ (úmido|seco), $\mathrm{D} \mid \mathrm{W}$ (seco|úmido) e $\mathrm{D} \mid \mathrm{D}$ (seco|seco). A partir dessas definições, a probabilidade de dia úmido seguido de dia úmido (Eq. 2) foi calculada segundo formulação de Sharpley \& Williams, 1990.

$$
\mathrm{P}(\mathrm{W} \mid \mathrm{W})=1.0-\beta+\mathrm{P}(\mathrm{W} \mid \mathrm{D})
$$

em que: $\mathrm{P}(\mathrm{W} \mid \mathrm{W})$ é a probabilidade de transição de dia chuvoso|chuvoso e $\mathrm{P}(\mathrm{W} \mid \mathrm{D})$ é a probabilidade de transição de dia chuvoso|seco.
Por definição, quando $\beta=1, P(W \mid D)=P(W \mid W)=P(W)$. Ao mesmo tempo, baixos valores de $\beta$ ocasionam efeitos sobre dias chuvosos e, se $\beta$ tende a zero, $\mathrm{P}(\mathrm{W} \mid \mathrm{D})$ tende a zero e $\mathrm{P}(\mathrm{W} \mid \mathrm{W})$ tende a 1 . Logo, observa-se que $\beta$ controla o intervalo entre eventos de chuva.

Modelos de temperatura máxima para dias secos e dias úmidos

Os modelos foram ajustados com base no princípio de que, tanto a radiação solar como a temperatura máxima do ar tendem a apresentar valores mais baixos em dias chuvosos se comparados a dias secos. Partindo-se de uma relação empírica entre o resultado de uma matriz e os erros, a fórmula de Richardson \& Nicks (1990) foi usada para obter o modelo da temperatura máxima (Eq. 3).

$$
\mathrm{TW}_{\mathrm{mx}, \mathrm{k}}=\mathrm{TD}_{\mathrm{mx}, \mathrm{k}}-\Omega_{\mathrm{T}}\left(\mathrm{T}_{\mathrm{mx}, \mathrm{k}}-\mathrm{T}_{\mathrm{mn}, \mathrm{k}}\right)
$$

em que: $\mathrm{TW}_{\mathrm{mx}, \mathrm{k}}$ é a temperatura máxima mensal para dias chuvosos $\left({ }^{\circ} \mathrm{C}\right)$ em um mês $\mathrm{k} ; \mathrm{TD}_{\mathrm{mx}, \mathrm{k}}$ é a temperatura máxima mensal para dias secos $\left({ }^{\circ} \mathrm{C}\right)$ em um mês $\mathrm{k}$; $\mathrm{T}_{\mathrm{mx}, \mathrm{k}}$ é a temperatura máxima mensal $\left({ }^{\circ} \mathrm{C}\right)$ em um mês $\mathrm{k}$; $\mathrm{T}_{\mathrm{mn}, \mathrm{k}}$ é a temperatura mínima mensal $\left({ }^{\circ} \mathrm{C}\right)$ para um mês k e $\Omega_{\mathrm{T}}$ é um fator de ajuste do modelo, que varia de 0,0 a 1,0 . Para $\Omega_{\mathrm{T}}=0$, ignoram-se os efeitos de dia úmido. De acordo com as condições climáticas testadas e apresentadas por Sharpley \& Williams $(1990), \Omega_{\mathrm{T}}$ varia entre 0,5 a 1,0 .

\section{Modelos de radiação solar para dias secos e dias úmidos}

O procedimento para a obtenção do modelo da radiação solar para dias chuvosos e secos foi similar ao da temperatura máxima e foi obtido como um ajuste a partir da radiação em dias secos conforme Sharpley \& Williams (1990), conforme Eq. 4.

$$
\mathrm{RAW}_{\mathrm{k}}=\Omega_{\mathrm{R}} \mathrm{RAD}_{\mathrm{k}}
$$

sendo: $\mathrm{RAW}_{\mathrm{k}}$ é a radiação solar mensal para dias chuvosos em um mês k em MJ m ${ }^{-2} ; \mathrm{RAD}_{k}$ é a radiação solar mensal para dias secos, em um mês k em MJ m ${ }^{-2}$ e $\Omega_{\mathrm{R}}$ é um fator de ajuste, que varia de 0 a 1,0 . Um valor de $\Omega_{R=} 0,5$ foi considerado satisfatório para atender a maioria dos locais avaliados por Sharpley \& Williams (1990).

Foram encontradas dificuldades na obtenção de dados de radiação solar a nível do solo para todos os locais e se adotou a fórmula proposta originalmente por Angström (1924) e citada por Pedro Júnior et al. (1989) para a estimativa da radiação solar, a fim de se comparar com os dados de radiação gerados pelos simuladores de dados meteorológicos do EPIC. A equação de Angström (1924) é função de horas de brilho solar (Eq. 5).

$$
\frac{\mathrm{R}_{\mathrm{s}}}{\mathrm{R}_{0}}=\mathrm{a}+\mathrm{b}\left(\frac{\mathrm{n}}{\mathrm{N}}\right)
$$

em que: $\mathrm{R}_{\mathrm{s}}$ é a radiação solar global a nível do solo; $\mathrm{R}_{0}$ é a radiação solar recebida no topo da atmosfera; a e b são constantes; $n$ é a duração do brilho solar (horas de insolação) e N é a duração máxima possível de brilho solar para determinada 
latitude. Os valores de radiação solar no topo da atmosfera foram obtidos em Salati et al. (1967).

Embora seja uma forma empírica de se obter a radiação solar, Pedro Júnior et al. (1989) discutiram a validade da utilização da equação de Angström (1924) adaptada posteriormente para as condições do Estado de São Paulo, por Cervellini et al. (1966). Há, no entanto, maior limitação no teste do modelo da radiação solar em virtude dessa aproximação.

\section{Ajuste dos modelos de precipitação, de temperatura máxima do ar e de radiação solar}

Organizou-se o banco de dados meteorológicos diários de chuva, temperaturas máximas e mínimas e radiação solar para cada localidade, em planilha Excel, a partir dos anos de 1962 a 1989 para o ajuste, e dos anos de 1990 a 1999 para o teste. Determinaram-se valores médios de $\beta, \Omega_{\mathrm{T}}$ e $\Omega_{\mathrm{R}}$ para cada mês do ano, a fim de se avaliar: 1) a variação sazonal desses fatores de ajuste; e 2) o desempenho devido à adoção de um valor médio para cada fator, conforme apresentado por Sharpley \& Williams (1990). Supõe-se que a simples aplicação desses valores representa uma aproximação excessiva, com possível redução da sensibilidade dos modelos.

Desta forma, foram testadas as Eqs. 1 a 4 utilizando-se, inicialmente, os valores propostos conforme a indicação de Sharpley \& Williams (1990) $\operatorname{com} \beta=0,75 ; \Omega_{\mathrm{T}}=0,75$ e $\Omega_{\mathrm{R}}=0,50$ referentes, respectivamente, aos modelos de precipitação, de temperatura máxima e de radiação solar para os municípiosteste. Paralelamente, calcularam-se valores de $\beta, \Omega_{\mathrm{T}}$ e $\Omega_{\mathrm{R}}$ para os mesmos locais se considerando, porém, a média anual obtida dos anos de 1962 a 1989 (ajuste), bem como se calcularam, também, os valores desses coeficientes para cada mês referente a esse mesmo período.

\section{Teste dos modelos}

Dados observados, não incluídos no ajuste dos modelos, foram a referência numérica dos testes dos resultados e utilizados, portanto, como dados independentes para a verificação do desempenho da previsão feita pelo modelo. Ao se correlacionar valores estimados com aqueles observados pela análise de regressão, pode-se considerar a precisão (r) e a concordância ou exatidão (d) como índices de validade das equações. A precisão, correspondente à dispersão dos valores em torno da média, é dada pelos coeficientes de correlação (r) ou de determinação $\left(\mathrm{R}^{2}\right)$. Conforme descrevem Camargo \& Camargo (2000), esses são índices estatísticos que indicam o grau de dispersão dos dados obtidos, ou seja, o erro aleatório. Os coeficientes de correlação poderão indicar alta precisão da estimativa, embora possibilitem apresentar-se vício de origem, com grande erro sistemático, podendo ainda resultar em informação precisamente errada.

A concordância refere-se à exatidão ou à aproximação dos dados estimados com os observados e pode ser avaliada graficamente, pelo afastamento dos pontos cotados no gráfico de regressão em relação à reta de valores iguais 1:1. Para quantificar matematicamente essa aproximação, foi desenvolvido por Willmott et al. (1985) um coeficiente designado concordância ou exatidão, representado pela letra d, cujos valores variam de 0 para nenhuma concordância e de 1,0 para concordância perfeita entre eles. O modelo pode ser representado pela Eq. 6 .

$$
\mathrm{d}=1-\left[\frac{\sum(\mathrm{Pi}-\mathrm{Oi})^{2}}{\sum(|\mathrm{Pi}-\mathrm{O}|+|\mathrm{Oi}-\mathrm{O}|)^{2}}\right]
$$

em que: Pi são os valores estimados; Oi são os valores observados e $\mathrm{O}$ é a média dos valores observados.

Outras medidas de desempenho do modelo são os erros sistemáticos (Es) e não-sistemáticos ou aleatórios (Ea), componentes da raiz quadrada do erro médio, e o erro médio absoluto (EAM), uma medida da magnitude entre as diferenças dos valores estimados e os reais.

\section{RESULTADOS E DISCUSSÃO}

\section{Resultado do ajuste do modelo de probabilidades para seqüência de dias secos e úmidos}

As equações dos modelos de probabilidades foram testadas para todos os locais, a partir dos dados diários (1962 a 1989) e se obtiveram valores de beta $(\beta)$ separadamente para cada mês e um valor médio anual (Tabela 1). É possível observar a variação sazonal de $\beta$ para todos os locais, como uma variável de distribuição inversa ao período de chuvas do Estado de São Paulo. O fator $\beta$ pondera as probabilidades de transição $\mathrm{P}(\mathrm{W} \mid \mathrm{W})$ e $\mathrm{P}(\mathrm{W} \mid \mathrm{D})$ em função da ocorrência ou não de chuvas e é naturalmente esperada, portanto, uma relação inversa com o comportamento e a distribuição da chuva. Observa-se, em alguns locais, queda mais acentuada de $\beta$ em relação à chuva, como acontece em Campinas, no mês de junho. Isso ocorre em função da diminuição da freqüência de dias com a transição úmido|seco (W|D) nesse mês e, como $\beta$ é diretamente proporcional a essa variação, ocorre também o decréscimo. Excetuando-se Ubatuba, os demais locais apresentaram picos de $\beta$ com valores próximos a 0,60 nos meses com baixa precipitação.

Ao se comparar os resultados dos diversos locais nota-se, primeiro, o valor anual baixo de beta para Ubatuba $(0,36)$ na Tabela 1, devido ao fato de Ubatuba apresentar chuva durante praticamente o ano todo, sem a estação de seca característica apresentada nas demais localidades. Como $\beta$ varia inversamente proporcional ao número de dias úmidos, esperam-se valores mais baixos.

Deve-se ressaltar que nenhum local apresentou valor de $\beta$ igual a 0,75 que, segundo as referências de Sharpley \& Williams (1990), seria um valor médio satisfatório conforme os testes realizados em diferentes localidades. Observa-se que o valor médio anual mais alto atingiu 0,51 em Campinas e Pindorama. Por outro lado, Pindorama apresentou os valores de $\beta$ mais altos, que correspondem aos períodos mais secos (maio a novembro). Esta situação evidencia a importância da escala temporal utilizada nesses modelos matemáticos, uma vez que médias e outras generalizações podem mascarar efeitos de eventos episódicos do clima. 
Tabela 1. Valores mensais e anuais de $\beta, \Omega_{\mathrm{T}}$ e $\Omega_{\mathrm{R}}$ referentes ao ajuste do modelo de probabilidades, da temperatura máxima do ar e da radiação solar, obtidos a partir da série de 1962 a 1989 para diferentes localidades

\begin{tabular}{|c|c|c|c|c|c|c|c|c|c|c|c|c|c|c|c|c|c|c|}
\hline \multirow[t]{2}{*}{ Mês } & \multicolumn{3}{|c|}{ Campinas } & \multicolumn{3}{|c|}{ Jaú } & \multicolumn{3}{|c|}{ Manduri } & \multicolumn{3}{|c|}{ Mococa } & \multicolumn{3}{|c|}{ Pindorama } & \multicolumn{3}{|c|}{ Ubatuba } \\
\hline & $(\beta)$ & $\left(\Omega_{\mathrm{T}}\right)$ & $\left(\Omega_{R}\right)$ & ( $\beta)$ & $\left(\Omega_{\mathrm{T}}\right)$ & $\left(\Omega_{R}\right)$ & $(\beta)$ & $\left(\Omega_{\mathrm{T}}\right)$ & $\left(\Omega_{\mathrm{R}}\right)$ & $(\beta)$ & $\left(\Omega_{\mathrm{T}}\right)$ & $\left(\Omega_{R}\right)$ & $(\beta)$ & $\left(\Omega_{\mathrm{T}}\right)$ & $\left(\Omega_{R}\right)$ & $(\beta)$ & $\left(\Omega_{\mathrm{T}}\right)$ & $\left(\Omega_{\mathrm{R}}\right)$ \\
\hline Janeiro & 0,36 & 0,15 & 0,75 & 0,36 & 0,22 & 0,75 & 0,34 & 0,16 & 0,79 & 0,27 & 0,25 & 0,74 & 0,35 & 0,22 & 0,79 & 0,29 & 0,27 & 0,76 \\
\hline Fevereiro & 0,44 & 0,22 & 0,77 & 0,33 & 0,22 & 0,78 & 0,33 & 0,18 & 0,77 & 0,33 & 0,24 & 0,75 & 0,32 & 0,20 & 0,81 & 0,25 & 0,25 & 0,71 \\
\hline Março & 0,47 & 0,19 & 0,77 & 0,47 & 0,18 & 0,78 & 0,44 & & 0,77 & 0,40 & 0,19 & & 0,38 & 0,21 & 0,80 & 0,31 & 0,25 & 0,72 \\
\hline Abril & 0,60 & 0,13 & 0,76 & 0,58 & 0,14 & & 0,55 & & 0 , & 0,53 & & & 0,59 & & 78 & 0,33 & 15 & 0,77 \\
\hline Maio & 0,56 & 0,21 & 0,69 & 0,56 & 0,18 & 0,70 & 0,48 & 0,21 & 0,67 & 0,64 & 0,19 & 0,75 & 0,63 & 0,25 & 0,71 & 0,44 & 0,22 & 0,69 \\
\hline Junho & 0,50 & 0,32 & 0,62 & 0,55 & 0,30 & 0,70 & 0,49 & 0,26 & 0,71 & 0,62 & 0,25 & 0,73 & 0,63 & 0,28 & 0,75 & 0,46 & 0,21 & 0,69 \\
\hline Julho & 0,66 & 0,34 & 0,75 & 0,63 & 0,33 & 0,77 & 0,63 & 0,33 & 0,77 & 0,64 & 0,28 & 0,72 & 0,62 & 0,38 & 0,73 & 0,46 & 0,18 & 0,67 \\
\hline Agosto & 0,65 & 0,38 & 0,72 & 0,59 & 0,38 & 0,69 & 0,54 & 0,32 & 0,71 & 0,59 & 0,34 & 0,72 & 0,65 & 0,34 & 0,74 & 0,47 & 0,32 & 0,65 \\
\hline Setembro & 0,53 & 0,35 & 0,72 & 0,51 & 0,39 & 0,73 & 0,49 & 0,34 & 0,71 & 0,54 & 0,32 & 0,76 & 0,59 & 0,33 & 0,77 & 0,40 & 0,31 & 0,77 \\
\hline Outubro & 0,51 & 0,29 & 0,71 & 0,49 & 0,29 & 0 , & 0,51 & 0,3 & 0,72 & 0,49 & 0 & 0,75 & 0,53 & 0,28 & 0,77 & 0,37 & 0,26 & 0,73 \\
\hline & 0,46 & 0,22 & 0,7 & 0,50 & 0,2 & & 0,51 & 0 & 0,7 & 0,39 & & 0 & 0,48 & 0,2 & 0,75 & 0,31 & 0,24 & 0,71 \\
\hline Dezen & 0,33 & 0,23 & 0,7 & 0,34 & 0,24 & 0 & 0,3 & 0,2 & 0,7 & 0,26 & 0,26 & 0,70 & 0,32 & 0,24 & 0,76 & 0,24 & 0,25 & 0,71 \\
\hline Anual & 0,51 & 0,26 & 0,72 & 0,49 & 0,26 & 0,74 & 0,47 & 0,24 & 0,73 & 0,48 & 0,25 & 0,74 & 0,51 & 0,26 & 0,76 & 0,36 & 0,24 & 0,72 \\
\hline
\end{tabular}

\section{Resultado do ajuste do modelo da temperatura máxima do ar}

A partir do banco de dados de temperatura máxima do ar, calcularam-se valores de $\Omega_{\mathrm{T}}$ em escala mensal e anual, seguindo-se o mesmo procedimento utilizado para o modelo de probabilidade.

Assim como $\beta, \Omega_{\mathrm{T}}$ representa um fator de ponderação entre as amplitudes das faixas de temperaturas máximas para dias secos e dias úmidos e das faixas de temperaturas máximas e mínimas, baseadas na ocorrência ou ausência de chuvas. Esta relação foi determinada a partir de um ajuste empírico baseado na matriz e nos erros, já referidos.

Na Tabela 1 estão apresentados os valores de $\Omega_{\mathrm{T}}$ para todos os locais, incluindo todos os meses do ano e a média anual. Uma simples análise mostra que em nenhum local se encontrou valor de $\Omega_{\mathrm{T}}$ médio entre 0,5 e 1,0 (Sharpley \& Williams, 1990). O valor máximo encontrado para $\Omega_{\mathrm{T}}$ foi $0,26 \mathrm{e}$, portanto, evidencia incertezas quanto ao desempenho do modelo ao se aplicar o intervalo recomendado para os locais paulistas.

Analisando-se, em escala crescente e conforme o modelo, nota-se que Manduri apresentou valor médio anual de $\Omega_{\mathrm{T}}$ igual ao de Ubatuba e isto pode ser explicado por ocorrer, nesse local, maior valor de amplitude térmica em relação a Ubatuba, já que $\Omega_{\mathrm{T}}$ é inversamente proporcional à amplitude. Por outro lado, Ubatuba apresenta menor valor de amplitude de faixa de temperatura, mas como a quantidade de dias úmidos é muito elevada, o valor de $\Omega_{\mathrm{T}}$ decresce.

Mococa apresentou valor intermediário anual de $\Omega_{\mathrm{T}}$ entre as localidades e, embora a amplitude da faixa de temperatura tenha sido bastante próxima da obtida nos demais locais, a diferença entre $\mathrm{TD}_{\mathrm{mx}}$ e $\mathrm{TW}_{\mathrm{mx}}$ é menor, resultando no valor 0,25 . Campinas, Jaú e Pindorama foram os locais que apresentaram valor mais alto para $\Omega_{\mathrm{T}}$ médio $(0,26)$, sendo observado um valor máximo em Pindorama, definido pelas casas decimais.

\section{Resultado do ajuste do modelo da radiação solar}

Avaliando-se a média anual de $\Omega_{\mathrm{R}}$ em ordem crescente (Tabela 1) Campinas e Ubatuba tiveram os valores mais baixos $(0,72)$; na seqüência está Manduri $(0,73)$; em seguida Jaú e Mococa, que apresentaram o mesmo valor de $\Omega_{\mathrm{R}}(0,74)$; por último, Pindorama apresentou o valor mais alto $(0,76)$. Nota-se que nenhum local apresentou o valor $\Omega_{\mathrm{R}}$ recomendado por Sharpley \& Williams (1990), igual a 0,5 . Todos os resultados para $\Omega_{\mathrm{R}}$ foram superiores a 0,70 .

Analisando-se os ajustes em escala mensal e se considerando que a variação de $\Omega_{\mathrm{R}}$ é diretamente proporcional à radiação solar para dias úmidos (Eq. 4), observa-se que os valores mais altos encontrados estão entre os meses de janeiro a abril para todos os locais com decréscimo no período de inverno, exceto Ubatuba, que também mostrou valor elevado em setembro (semelhante ao mês de abril), correspondendo aos resultados apresentados anteriormente por Pedro Júnior et al. (1989). Da mesma forma, Campinas apresentou valor baixo para $\Omega_{\mathrm{R}}$ no mês de junho, porém este resultado também se apresenta coerente com os dados apresentados por Pedro Júnior et al. (1989), em que se observa situação semelhante para esta localidade e no mesmo mês.

Jaú apresentou elevado $\Omega_{\mathrm{R}}$ em julho, mas, ao conferir o banco de dados, observou-se aumento no valor da radiação solar para dias úmidos, como a provável causa desse resultado. Ao se comparar os meses de abril e maio, constatou-se que Manduri apresentou os valores mais baixos, fato este resultado da menor amplitude entre a radiação para dias úmidos e para dias secos presentes nos dados históricos dessa localidade.

Para os meses de fevereiro e março, Pindorama teve os valores mais altos de $\Omega_{\mathrm{R}}$ em dias úmidos, como conseqüência provável de sua variação de proporcional à radiação. Em Ubatuba notou-se uma característica de aumento no valor da radiação em setembro, comparado aos meses de agosto e outubro.

\section{Resultado do teste dos modelos de probabilidades}

Os resultados do teste de concordância (d) para os modelos $\mathrm{P}(\mathrm{W} \mid \mathrm{D})$ e $\mathrm{P}(\mathrm{W} \mid \mathrm{W})$ para Campinas, Jaú, Manduri, Mococa, Pindorama e Ubatuba, estão apresentados nas Tabelas 2 a 4. Os testes foram realizados para valores de $\beta, \Omega_{\mathrm{T}}$ e $\Omega_{\mathrm{R}}$, determinados para cada mês, para a média anual e para os valores recomendados por Sharpley \& Williams (1990).

Analisando-se, inicialmente, o desempenho do modelo para a estimativa de $\mathrm{P}(\mathrm{W} \mid \mathrm{D})$, nota-se que, ao se utilizar $\beta=0,75$, o desempenho foi pior em relação aos modelos com valores de $\beta$ 
mensais e anual. Mesmo quando ocorre alto valor de $\mathrm{R}^{2}$, observam-se baixo índice de concordância e valores elevados de erros. Para as condições do Estado de São Paulo, os valores encontrados de $\beta$ permaneceram no intervalo entre 0,47 e 0,51 .

Em Campinas, o melhor desempenho foi para $\beta$ anual, com $\mathrm{R}^{2}=0,78$ e $\mathrm{d}=0,85$ e, ao se aplicar $\beta$ mensal, as estimativas decresceram. Jaú e Mococa indicaram boas estimativas para $\mathrm{P}(\mathrm{W} \mid \mathrm{D})$, com melhor resultado para $\beta$ mensal em ambos os casos, enquanto em Pindorama o desempenho foi bom para o modelo nas duas situações de $\beta$. Ubatuba, entretanto, apresentou pior desempenho entre todas as localidades e não houve correlação entre dados observados e estimados para $\beta$ mensal. Neste local, não foi possível aplicar o modelo de P(W|D) nas condições de ajuste. Pode-se atribuir, no entanto, ao fato de Ubatuba praticamente não apresentar estação seca durante o ano e, neste caso, o limiar de $0,2 \mathrm{~mm}$ recomendado por Sharpley \& Williams (1990) pode estar muito baixo para essas condições de regime de chuva. Da mesma forma, como o total de chuva durante o ano é elevado em Ubatuba, a situação pode mascarar a freqüência de $\mathrm{P}(\mathrm{W} \mid \mathrm{D})$ para a determinação de $\beta$.

Para as estimativas de $\mathrm{P}(\mathrm{W} \mid \mathrm{W})$, o comportamento foi semelhante ao das estimativas $\operatorname{com} \beta$ igual a 0,75 e para $\beta$ anual em quase todos os locais. Notou-se que, para os casos onde se adotou 0,75 , o desempenho foi satisfatório, ou seja, melhor do que as estimativas para $\mathrm{P}(\mathrm{W} \mid \mathrm{D})$. Este resultado talvez possa ser atribuído ao fato desses modelos apresentarem

Tabela 2. Resultados do teste de concordância de Willmott referentes aos modelos $\mathrm{P}(\mathrm{W} \mid \mathrm{D})$ e $\mathrm{P}(\mathrm{W} \mid \mathrm{W})$ para todas as localidades, com os respectivos coeficientes de determinação $\left(\mathrm{R}^{2}\right)$ e erros médio absoluto (EMA), sistemático (Es) e aleatório (Ea)

\begin{tabular}{|c|c|c|c|c|c|c|c|c|c|c|}
\hline Modelos de Probabilidades & $\mathrm{R}^{2}$ & d & EMA $^{*}$ & $\mathrm{Es}^{*}$ & $\mathrm{Ea}^{*}$ & $\mathrm{R}^{2}$ & d & EMA $^{*}$ & $\mathrm{Es}^{*}$ & $\mathrm{Ea}^{*}$ \\
\hline & \multicolumn{5}{|c|}{ Campinas } & \multicolumn{5}{|c|}{ Mococa } \\
\hline $\mathrm{P}(\mathrm{W} \mid \mathrm{D}) \beta$ anual & 0,78 & 0,85 & 0,04 & 0,04 & 0,03 & 0,85 & 0,89 & 0,03 & 0,03 & 0,03 \\
\hline $\mathrm{P}(\mathrm{W} \mid \mathrm{W}) \beta$ anual & 0,62 & 0,31 & 0,20 & 0,03 & 0,24 & 0,74 & 0,33 & 0,23 & 0,03 & 0,28 \\
\hline $\mathrm{P}(\mathrm{W} \mid \mathrm{D}) \beta$ mensal & 0,53 & 0,85 & 0,03 & 0,04 & 0,01 & 0,92 & 0,97 & 0,01 & 0,01 & 0,01 \\
\hline $\mathrm{P}(\mathrm{W} \mid \mathrm{W}) \beta$ mensal & 0,39 & 0,55 & 0,18 & 0,05 & 0,19 & 0,85 & 0,63 & 0,21 & 0,04 & 0,22 \\
\hline $\mathrm{P}(\mathrm{W} \mid \mathrm{D}) \beta \quad 0,75$ & 0,79 & 0,59 & 0,09 & 0,06 & 0,11 & 0,85 & 0,59 & 0,11 & 0,06 & 0,13 \\
\hline \multirow[t]{2}{*}{$\mathrm{P}(\mathrm{W} \mid \mathrm{W}) \beta 0,75$} & 0,62 & 0,15 & 0,15 & 0,03 & 0,18 & 0,74 & 0,10 & 0,17 & 0,03 & 0,20 \\
\hline & \multicolumn{5}{|c|}{ Jaú } & \multicolumn{5}{|c|}{ Pindorama } \\
\hline $\mathrm{P}(\mathrm{W} \mid \mathrm{D}) \beta$ anual & 0,89 & 0,92 & 0,03 & 0,03 & 0,02 & 0,90 & 0,89 & 0,03 & 0,03 & 0,04 \\
\hline $\mathrm{P}(\mathrm{W} \mid \mathrm{W}) \beta$ anual & 0,78 & 0,31 & 0,21 & 0,02 & 0,25 & 0,82 & 0,31 & 0,22 & 0,02 & 0,26 \\
\hline $\mathrm{P}(\mathrm{W} \mid \mathrm{D}) \beta$ mensal & 0,92 & 0,97 & 0,01 & 0,01 & 0,01 & 0,90 & 0,97 & 0,01 & 0,01 & 0,01 \\
\hline $\mathrm{P}(\mathrm{W} \mid \mathrm{W}) \beta$ mensal & 0,71 & 0,55 & 0,20 & 0,03 & 0,21 & 0,89 & 0,61 & 0,20 & 0,03 & 0,20 \\
\hline $\mathrm{P}(\mathrm{W} \mid \mathrm{D}) \beta 0,75$ & 0,88 & 0,62 & 0,09 & 0,04 & 0,11 & 0,90 & 0,65 & 0,09 & 0,04 & 0,11 \\
\hline \multirow[t]{2}{*}{$\mathrm{P}(\mathrm{W} \mid \mathrm{W}) \beta 0,75$} & 0,78 & 0,15 & 0,13 & 0,02 & 0,16 & 0,82 & 0,09 & 0,14 & 0,02 & 0,17 \\
\hline & \multicolumn{5}{|c|}{ Manduri } & \multicolumn{5}{|c|}{ Ubatuba } \\
\hline $\mathrm{P}(\mathrm{W} \mid \mathrm{D}) \beta$ anual & 0,63 & 0,88 & 0,03 & 0,04 & 0,01 & 0,25 & 0,48 & 0,03 & 0,04 & 0,01 \\
\hline $\mathrm{P}(\mathrm{W} \mid \mathrm{W}) \beta$ anual & 0,52 & 0,34 & 0,22 & 0,03 & 0,25 & 0,13 & 0,44 & 0,16 & 0,01 & 0,19 \\
\hline $\mathrm{P}(\mathrm{W} \mid \mathrm{D}) \beta$ mensal & 0,42 & 0,73 & 0,03 & 0,03 & 0,04 & - & - & - & - & - \\
\hline $\mathrm{P}(\mathrm{W} \mid \mathrm{W}) \beta$ mensal & 0,49 & 0,57 & 0,20 & 0,05 & 0,21 & 0,48 & 0,57 & 0,16 & 0,05 & 0,17 \\
\hline $\mathrm{P}(\mathrm{W} \mid \mathrm{D}) \beta \quad 0,75$ & 0,60 & 0,63 & 0,08 & 0,06 & 0,08 & 0,24 & 0,09 & 0,20 & 0,08 & 0,21 \\
\hline $\mathrm{P}(\mathrm{W} \mid \mathrm{W}) \beta 0,75$ & 0,52 & 0,26 & 0,13 & 0,03 & 0,19 & 0,23 & 0,39 & 0,23 & 0,01 & 0,26 \\
\hline
\end{tabular}

* Adimensionais

Tabela 3. Resultados do teste de concordância de Willmott referentes ao modelo da temperatura máxima do ar (Mtmáx) para dias úmidos (Tw) para todas as localidades, com os respectivos coeficientes de determinação $\left(\mathrm{R}^{2}\right)$ e erros médio absoluto (EMA), sistemático (Es) e aleatório (Ea)

\begin{tabular}{|c|c|c|c|c|c|c|c|c|c|c|}
\hline Mtmáx & $\mathrm{R}^{2}$ & $\mathrm{~d}$ & EMA $^{*}$ & $\mathrm{Es}^{*}$ & $\mathrm{Ea}^{*}$ & $\mathrm{R}^{2}$ & d & EMA $^{*}$ & $\mathrm{Es}^{*}$ & $\mathrm{Ea}^{*}$ \\
\hline & \multicolumn{5}{|c|}{ Campinas } & \multicolumn{5}{|c|}{ Mococa } \\
\hline $\mathrm{T}(\mathrm{w}) \Omega_{\mathrm{T}}$ anual & 0,88 & 0,96 & 0,74 & 0,78 & 0,49 & 0,89 & 0,96 & 0,78 & 0,68 & 0,55 \\
\hline $\mathrm{T}(\mathrm{w}) \Omega_{\mathrm{T}}$ mensal & 0,91 & 0,98 & 0,60 & 0,83 & 0,05 & 0,97 & 0,98 & 0,44 & 0,39 & 0,36 \\
\hline \multirow[t]{2}{*}{$\mathrm{T}(\mathrm{w}) \Omega_{\mathrm{T}} 0,75$} & 0,86 & 0,57 & 5,18 & 0,97 & 5,18 & 0,95 & 0,53 & 5,96 & 0,57 & 5,96 \\
\hline & & & Jaú & & & \multicolumn{5}{|c|}{ Pindorama } \\
\hline $\mathrm{T}(\mathrm{w}) \Omega_{\mathrm{T}}$ anual & 0,96 & 0,96 & 0,81 & 0,45 & 0,79 & 0,87 & 0,96 & 0,70 & 0,80 & 0,50 \\
\hline $\mathrm{T}(\mathrm{w}) \Omega_{\mathrm{T}}$ mensal & 0,94 & 0,96 & 0,88 & 0,69 & 0,80 & 0,93 & 0,98 & 0,48 & 0,70 & 0,05 \\
\hline \multirow[t]{2}{*}{$\mathrm{T}(\mathrm{w}) \Omega_{\mathrm{T}} 0,75$} & 0,95 & 0,52 & 6,38 & 0,55 & 6,39 & 0,94 & 0,54 & 6,12 & 0,64 & 6,12 \\
\hline & \multicolumn{5}{|c|}{ Manduri } & \multicolumn{5}{|c|}{ Ubatuba } \\
\hline $\mathrm{T}(\mathrm{w}) \Omega_{\mathrm{T}}$ anual & 0,96 & 0,96 & 0,96 & 0,53 & 0,93 & 0,91 & 0,96 & 0,74 & 0,72 & 0,78 \\
\hline $\mathrm{T}(\mathrm{w}) \Omega_{\mathrm{T}}$ mensal & 0,96 & 0,98 & 0,80 & 0,62 & 0,77 & 0,88 & 0,95 & 0,94 & 0,85 & 0,77 \\
\hline $\mathrm{T}(\mathrm{w}) \Omega_{\mathrm{T}} 0,75$ & 0,94 & 0,53 & 7,21 & 0,79 & 7,21 & 0,84 & 0,66 & 4,13 & 1,09 & 4,16 \\
\hline
\end{tabular}


Tabela 4. Resultados do teste de concordância de Willmott referentes ao modelo da radiação solar (MRsolar) para dias úmidos $(\mathrm{Rw})$ para todas as localidades, com os respectivos coeficientes de determinação $\left(\mathrm{R}^{2}\right)$ e erros médio absoluto (EMA), sistemático (Es) e aleatório (Ea)

\begin{tabular}{|c|c|c|c|c|c|c|c|c|c|c|}
\hline MRsolar & $\mathrm{R}^{2}$ & $\mathrm{~d}$ & EMA $^{*}$ & $\mathrm{Es}^{*}$ & $\mathrm{Ea}^{*}$ & $\mathrm{R}^{2}$ & $\mathrm{~d}$ & EMA $^{*}$ & $\mathrm{Es}^{*}$ & $\mathrm{Ea}^{*}$ \\
\hline & \multicolumn{5}{|c|}{ Campinas } & \multicolumn{5}{|c|}{ Mococa } \\
\hline $\mathrm{R}(\mathrm{W}) \Omega_{\mathrm{R}}$ Anual & 0,89 & 0,96 & 0,84 & 0,88 & 0,63 & 0,92 & 0,96 & 0,87 & 0,77 & 0,73 \\
\hline $\mathrm{R}(\mathrm{W}) \Omega_{\mathrm{R}}$ Mensal & 0,90 & 0,97 & 0,79 & 0,92 & 0,32 & 0,86 & 0,95 & 1,06 & 1,00 & 0,75 \\
\hline \multirow[t]{2}{*}{$\mathrm{R}(\mathrm{W}) \Omega_{\mathrm{R}} 0,50$} & 0,89 & 0,59 & 4,95 & 0,60 & 5,10 & 0,91 & 0,56 & 5,51 & 0,54 & 5,64 \\
\hline & & & Jaú & & & \multicolumn{5}{|c|}{ Pindorama } \\
\hline $\mathrm{R}(\mathrm{W}) \Omega_{\mathrm{R}}$ Anual & 0,94 & 0,97 & 0,88 & 0,59 & 0,85 & 0,85 & 0,96 & 0,83 & 1,07 & 0,23 \\
\hline $\mathrm{R}(\mathrm{W}) \Omega_{\mathrm{R}}$ Mensal & 0,93 & 0,97 & 0,73 & 0,75 & 0,62 & 0,84 & 0,95 & 1,03 & 1,26 & 0,25 \\
\hline \multirow[t]{2}{*}{$\mathrm{R}(\mathrm{W}) \Omega_{\mathrm{R}} 0,50$} & 0,94 & 0,56 & 5,58 & 0,43 & 5,78 & 0,85 & 0,55 & 5,38 & 0,71 & 5,49 \\
\hline & \multicolumn{5}{|c|}{ Manduri } & \multicolumn{5}{|c|}{ Ubatuba } \\
\hline $\mathrm{R}(\mathrm{W}) \Omega_{\mathrm{R}}$ Anual & 0,89 & 0,90 & 1,50 & 0,85 & 1,71 & 0,82 & 0,92 & 1,24 & 1,06 & 1,12 \\
\hline $\mathrm{R}(\mathrm{W}) \Omega_{\mathrm{R}}$ Mensal & 0,84 & 0,92 & 1,38 & 1,13 & 1,44 & 0,70 & 0,90 & 1,40 & 1,50 & 1,08 \\
\hline $\mathrm{R}(\mathrm{W}) \Omega_{\mathrm{R}} 0,50$ & 0,88 & 0,57 & 5,73 & 0,58 & 6,06 & 0,82 & 0,59 & 4,60 & 0,73 & 4,85 \\
\hline
\end{tabular}

comportamento inverso no que se refere às probabilidades de transição $\mathrm{P}(\mathrm{W} \mid \mathrm{D})$ e $\mathrm{P}(\mathrm{W} \mid \mathrm{W})$. A partir dos resultados do modelo $\mathrm{P}(\mathrm{W} \mid \mathrm{W})$ para as condições anuais e mensais, é possível verificar que as duas condições apresentaram comportamento diferenciados, o que leva à suposição de que, para este modelo, outros fatores além da ocorrência de chuva podem interferir, como as probabilidades $\mathrm{P}(\mathrm{D} \mid \mathrm{W})$.

\section{Resultado do teste do modelo da temperatura do ar}

De forma semelhante ao modelo da chuva observa-se, para todos os locais sem exceção, que o pior desempenho encontrado foi para $\Omega_{\mathrm{T}}$ igual a 0,75 (Sharpley \& Williams, 1990). Notase, também, nos dados da Tabela 3 que os valores de $\mathrm{R}^{2}$ são altos e o índice d se encontra na média de 0,55 , porém, os erros das estimativas são muito elevados, comprometendo a eficácia do modelo. Em Manduri, por exemplo, o erro médio absoluto (EMA) e o erro absoluto (Ea) atingiram valores superiores a $7,0^{\circ} \mathrm{C}$.

No caso das estimativas para $\Omega_{\mathrm{T}}$ mensal e anual, obteve-se boa correlação entre os dados observados e os estimados em todas as localidades, com valores de $\mathrm{R}^{2} \mathrm{e}$ índice $\mathrm{d}$ superiores a 0,85 para ambos, respectivamente. Ubatuba apresentou melhor resultado quando comparado ao modelo da precipitação. Entre as localidades, Ubatuba foi a que teve desempenho inferior para $\Omega_{\mathrm{T}}$ mensal, mas ainda com altos $\mathrm{R}^{2} \mathrm{e}$ índice d.

\section{Resultado do teste do modelo da radiação solar}

Constatou-se, para todas as localidades, que o pior desempenho do modelo da radiação solar foi para o que se adotou $\Omega_{\mathrm{R}}$ igual a 0,50 (Sharpley \& Williams, 1990), conforme resultados descritos na Tabela 4. Quanto ao desempenho dos modelos para $\Omega_{\mathrm{R}}$ anual e mensal, observa-se que não há diferença significativa entre ambos, no que se refere aos coeficientes de determinação $\left(R^{2}\right)$ e ao índice d; entretanto, ressaltam-se os casos de Manduri e Ubatuba, que apresentaram erros superiores a $1,0 \mathrm{MJ} \mathrm{m}^{-2}$ para as estimativas com $\Omega_{\mathrm{R}}$ mensal e anual.

\section{CONCLUSÕES}

1. Os melhores resultados encontrados para os modelos testados referem-se à aplicação dos fatores de ajuste determinados a partir da série histórica de dados.

2. Constatou-se bom desempenho para os valores de $\beta$ encontrados nos ajustes mensal e anual para o modelo $\mathrm{P}(\mathrm{W} \mid \mathrm{D})$ a partir da série de dados de chuva em cada local, exceto Ubatuba.

3. Constatou-se um comportamento diferenciado para as escalas mensais e anuais no que se refere ao modelo de estimativa de $\mathrm{P}(\mathrm{W} \mid \mathrm{W})$, com desempenho inferior ao de $\mathrm{P}(\mathrm{W} \mid \mathrm{D})$ para todos os locais.

4. Os modelos da temperatura máxima do ar e da radiação solar são aplicáveis para São Paulo, com melhores resultados a partir dos ajustes.

\section{AGRADECIMENTOS}

Ao Conselho Nacional de Desenvolvimento Científico e Tecnológico (CNPq) e à Coordenação de Aperfeiçoamento de Pessoal de Nível Superior (CAPES) pelo apoio financeiro.

\section{LITERATURA CITADA}

Angström, A. Solar and terrestrial radiation. Quarterly Journal of the Royal Meterological Society, London, v.50, p.121126, 1924.

Camargo, A.P.; Camargo, M.B.P. Uma revisão analítica da evapotranspiração potencial. Bragantia, Campinas, v.59, n.2, p.125-137, 2000.

Cervellini, A.; Salati, E.; Godoy, H. Estimativas da distribuição da energia solar no Estado de São Paulo. Bragantia, Campinas, v.25, n.3, p.31-40, 1966.

Cooter, E.J.; Dhakhwa, G.B.A solar radiation model for use in biological applications in the south and southeastern USA. Agricultural and Forest Meteorology, Amsterdam, v.78, p.3135, 1995. 
Easterling, W.E.; Chen, X.; Hays, C.J.; Brandle, J.; Zhang, H. Improving the validation of model-simulated crop yield response to climate change: an application to the EPIC model. Climate Research, Oldendorf, v.6, n.3, p.263-273, 1996.

Gabriel, K.R.; Neumann, J. A. Markov chain for rainfall occurrence at Tel Aviv. Quarterly Journal of the Royal Meteorological Society, London, v.88 n. 375, p.90-95, 1962.

Nicks, A.D. Stochastic generation of the occurrence, pattern, and location of maximum mount of daily rainfall. In: Proceedings Symposium on Statistical Hydrology. Tucson. 1974. p.154-171, Publication No.1275

Pedro Júnior, M.J.; Alfonsi, R.R.; Camargo, M.B.P.; Chiavegatto, O.M.D.P.; Ortolani, A.A .; Brunini, O. Disponibilidade de radiação solar global para o Estado de São Paulo. Campinas: IAC, 1989. 13p. Boletim Técnico n. 123

Rao, M.N.; Waits, D.A.; Mitchell, L.N. A GIS-based modeling approach for implementation of sustainable farm management practices. Environmental Modelling \& Software, Canberra, v.15, n. 8, p.745-753, 2000.

Richardson, C.W. Stochastic simulation of daily precipitation, temperature and solar radiation. Water Resources Research, New York, v.17, n.1, p.182-190, 1981.

Richardson, C.W. Dependence structure of daily temperature and solar radiation. Transactions of the ASAE, St. Joseph, v.25, n. 3, p.735-739, 1982.

Richardson, C.W.; Nicks, A.D. Weather generator description. In: Erosion/productivity impact calculator. Model documentation. Washington, D.C: USDA-ARS Techinical, 1990. 235p. Bulletin 1768
Salati, E.; Cervellini, A.; Villa Nova, N.A. Estimativa da radiação solar que atinge uma área horizontal unitária admitindo-se a ausência da atmosfera. Rio de Janeiro: Serviço de Meteorologia, 1967, 57p. Boletim Técnico 6

Sansigolo, C.A. Modelagem estocástica de precipitações diárias. Revista Brasileira de Engenharia, Caderno de Recursos Hídricos, Porto Alegre, v.6, n.1, p.29-41, 1988.

Sentelhas, P.C.; Faria, R.T.; Chaves, M.O.; Hoogenboom, G.. Evaluation of the WGEN and SIMMETTEO weather generators for the brazilian tropics and subtropics, using crop simulation models. Revista Brasileira de Agrometeorologia, Santa Maria, v.9, n.2, p.357-376, 2001.

Sharpley, A.N.; Williams, J.R. Erosion/productivity impact calculator. Model documentation. Washington, D.C: USDAARS Techinical , 1990. 235p. Bulletin 1768

Stockle, C.D.; Williams, J. R.; Rosenberg, N.J.; Jones, C.A. A method for estimating direct and climatic effects of rising atmosferic carbon dioxide on growth and yield of crops: Part I. Modification of the EPIC model for climate change analysis. Agricultural Systems, New York, v.38, n.3, p.225238, 1992.

Virgens Filho, J.S.; Cataneo, A. Modelo computacional para simulação de radiação solar diária. Energia na Agricultura, Botucatu, v.14, n.2, p.26-36, 1999.

Willmott, C.J.; Ackleson, S.G.; Davis, J.J. Feddema, K. M.; Klink, D. R. Statistics for the evaluation and comparison of models. Journal of Geophysical Research, Ottawa, v.90, n.5, p.89959005, 1985 .

Wischmeier, W.H.; Smith, D.D. Predicting rainfall erosion losses: a guide to conservation planning. Washington: USDA, 1978. 58 p. Agricultural Handbook, 537 